\title{
Severe Rhino-orbital Mucormycosis Infection of an Intensive Care Unit Patient: A Case Report
}

\section{Ayşe Şahin Tutak ${ }^{1 *}$, Hüseyin Avni Fındıklı1 , Sefer Aslan ${ }^{1}$, Bilge Aydın Türk², Mehmet Şirik ${ }^{3}$ and Garip Bekfilavioğlu'}

${ }^{1}$ Department of İnternal Medicine, Adıyaman University of Medical Faculty, Adıyaman, Turkey

${ }^{2}$ Department of Pathology, Faculty of Medicine, Adıyaman University, Adiyaman, Turkey

${ }^{3}$ Department of Radiology, Adiyaman University of Medical Faculty, Adiyaman, Turkey

"Corresponding Authors: Ayșe Şahin Tutak, Department of İnternal Medicine, Adıyaman University of Medical Faculty, Adiyaman, Turkey, Tel: +904162161015; Fax:+904162161015; E-Mail: aysesahintutak@hotmail.com

Received: 27 November 2017; Accepted: 08 December 2017; Published: 15 December 2017

\begin{abstract}
Mucormycosis is an opportunistic fungal infection, which is occasionally seen in immunosuppressive patients, and has high morbidity and mortality rates despite treatment. The case is here reported of a patient admitted to our Intensive Care Unit (ICU) with diagnosis of diabetic ketoacidosis, acute kidney injury, acute cerebral infarct and rhino-orbital mucormycosis. This level of advanced rhino-orbital mucormycosis caused severe cutaneous and subcutaneous lesions has not been reported previously in literature and thus the case is evaluated in respect of the early diagnosis of mucormycosis and treatment.
\end{abstract}

Keywords: Mucormycosis; Acute kidney injury; Rhinocerebral; Cavernous sinus syndrome; Diabetes mellitus

\section{Introduction}

Mucormycosis is the common name given to diseases caused by the Mucorales order of fungi, particularly mucor, rhizopus and absidia, which show a clinical course in the form of organ involvement. Mucorales are saprobic soil organisms, the majority of which are found on rotting organic material and are endemic throughout the world. These 
organisms become opportunistically pathogenic in individuals with a suppressed immune system, such as in diabetes and ketoacidosis, and can cause acute angio-invasive infections [1,2].

Mucormycosis can be seen in 6 different forms as rhinocerebral, pulmonary, central nervous system (CNS), gastrointestinal, subcutaneous and occasionally in a disseminated form. Rhinocerebral mucormycosis is the most commonly encountered form and is examined in 3 subgroups of rhino-maxillary, rhino-orbital and rhinoorbitocerebral. Generally the findings and symptoms of rhino-cerebral mucormycosis (RCM) are headache, rhinorrhea, intranasal or intraoral black necrotic areas and epistaxis. As the disease progresses, orbital cellulitis, orbital apex syndrome, cavernous sinus syndrome and CNS involvement may be observed [3].

\section{Case}

A 61-year old male was found unconscious at home and was brought to the Emergency Department. According to information from the family, he lived alone most of the time despite being a patient needing nursing care. The medical records revealed a diagnosis of diabetes mellitus. At the time of admittance, the patient was unconscious, there were scattered necrotic scars on the left eye and periorbital area, temperature was $39.2^{\circ} \mathrm{C}$, blood pressure $75 / 50$ $\mathrm{mm} \mathrm{Hg}$, pulse 113 beats/minute, and respiratory rate was 19 breaths/minute with oxygen saturation of $86 \%$ on room air. The patient was admitted to the Internal Medicine ICU. The results of the hematological examination on admittance were $\mathrm{Hb}$ (hemoglobin): $8.5 \mathrm{~g} / \mathrm{dL}$, Htc(hematocrit): 28.3\%, WBC (white blood cell count) 21,000/mm, Plt (platelet count): $72 \times 104 / \mathrm{mm}^{3}$. Urinalysis showed keton ++ , blood chemistry test results showed an elevated blood glucose level of $580 \mathrm{mg} / \mathrm{dL}$, creatinine $4.2 \mathrm{mg} / \mathrm{dL}$, urea: $158 \mathrm{mg} / \mathrm{dl}$, sodium $128 \mathrm{mg} / \mathrm{dL}$, potassium $5.9 \mathrm{mg} / \mathrm{dL}$, arterial blood gas analysis; $\mathrm{pH} 7.16, \mathrm{HCO}_{3}: 12$.

As a result of the biochemical tests, the patient was evaluated as acute renal failure (ARF) and diabetic ketoacidosis (DCA). On the requested magnetic resonance imaging (MRI), a wide area compatible with the area irrigated by the left middle cerebral artery (MCA) was evaluated as acute infarct (Figure 1A and 1B), and widespread tissue necrosis was observed in the rhino-orbital region (Figure 2) was consistent with rhino-orbital mucormycosis (ROM). Biopsy material was taken from the necrotic areas and sent to the microbiology laboratory for histopathological examination. The microscopic examination of the biopsy material showed hyphae which were pleomorphic, irregular, thick and branching without septa within the characteristic squamous epithelium and keratin (Figure 3). Treatment was started of intravenous insulin infusion and Amphotericin B $0.3 \mathrm{mg} / \mathrm{kg} / \mathrm{day}$ and because of the impaired kidney function, there could be no staged dose increase. As the patient had thrombocytopenia and was receiving anticoagulant treatmnt, a limited amount of necrotic tissue debridement could be made. During the followup, the DCA recovered and VE ARF partially regressed and the patient was accepted as ex on the 14th day after admission. 

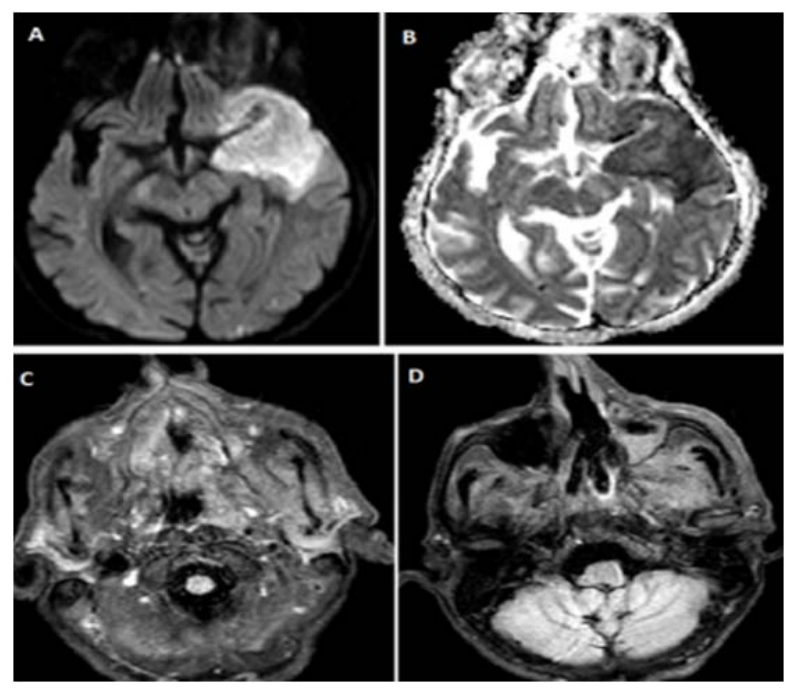

Figure 1 (A, B, C, D): On diffusion weighted images, acute ischaemia with compatible diffusion restriction observed in the left temporal lobe. (A, B). On axial FLAIR images, air-fluid levelling in the left maxillary sinus and hyperintense signal changes were observed compatible with intense oedema secondary to infection in the superficial and deep soft tissue planes in the left hemifacial area (C, D).

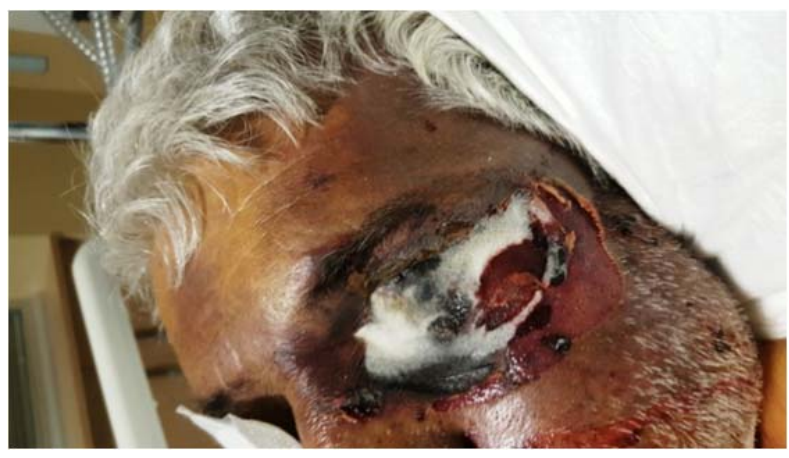

Figure 2: Image showing the tissue necrosis and mold was observed in the rhino-orbital region.

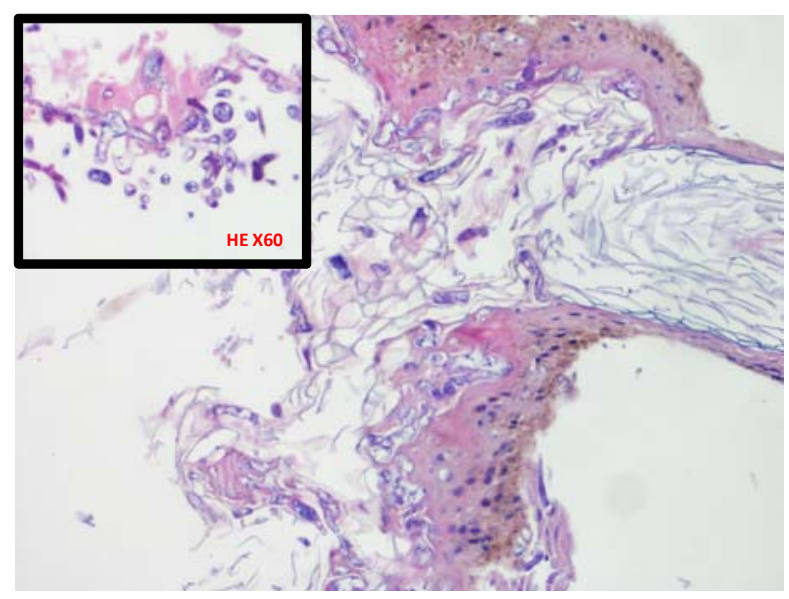

Figure 3: On the haematoxylin and eosin stained preparate (x40-50), hyphae which were pleomorphic, irregular, 


\section{Discussion}

Mucormycosis is an opportunistic infection that emerges in immune-compromised patients. Predisposing factors for mucormycosis include uncontrolled diabetes (especially ketoacidosis patients), malignancies such as lymphoma and leukaemia, renal failure, organ transplantations, long-term corticosteroid and immunosuppressive treatment, cirrhosis, protein energy malnutrition and AIDS. Mucormycosis often affects patients with uncontrolled diabetes, and of reported rhino-cerebral mucormycosis cases, $88 \%$ have been determined with uncontrolled diabetes and of those, 53.3\% with diabetic ketoacidosis [4-7]. The mechanisms held responsible for the more frequent occurrence of mucormycosis in diabetic patients are the reduced iron-binding capacity of transferrin in this patient group and the rapid production of fungi in ketoacidotic and/or hyperglycemic conditions with fungi more easily using iron in the serum to be able to reproduce more quickly, thereby creating a vicious circle with the increased numbers of fungi making more vascular invasion and increasing ischaemia and acidosis [8]. In the currrent case, the predisposing factors for ROM were found together (diabetes, DCA, acute renal failure).

As the reported mortality rate for mucormycosis has been stated in a wide range of $50 \%-100 \%$, this rate can of course be changed positively with early diagnosis [9]. In the diagnosis of the disease, histopathology or culture are accepted as the gold standard [10]. In the treatment of disease showing an agressive and mortal course, it is recommended that the underlying disease is brought under control, antifungal treatment is administered in addition to surgical debridement, and Amphotericin B is recommended as the prmary antifungal treatment [11]. In the current patient, by providing blood glucose regulation, the DCA was improved and conventional Amphotericin B treatment was started at the same time as the application of surgical debridement. As the disease had a known aggressive and mortal course and because of the comorbidities of ischaemic stroke and acute renal failure, the patient was ex on the 15th day of hospitalisation.

The majority of Mucorales group fungi spread as an agressive focus of infection from the paranasal sinuses to the orbital or cranial area. As they are naturally angiophillic in character, this can lead to early invasion of blood vessels, vascular occlusion, tissue necrosis and ischaemia or haemorrhage [12]. In the current patient, widespread tissue necrosis was observed in the rhino-orbital region, and necrotic scarring filling the intranasal area, the orbital cavity and the sinuses, which was associated with tissue necrosis developing after vascular invasion (Figure 1C, 1D and Figure 2). Although very few cases have been reported in literature [13, 14], even if there are cases of mucormycosis which developed cerebral infarct because of vascular invasion, in the current case there were no cerebral imaging findings or findings of cerebral vascular invasion which could show a reason for the acute ischaemic stroke.

It must be stated that ROM, which is seen in the diabetic patient group in particular, could be a reflection of poor diabetic control, poor socio-economic factors, patient education and low awareness. This case was presented with the aim of reminding that mucormycosis is an opportunistic infection with high morbidity and mortality and of creating a high index of suspicion in clinicians for individuals who have predisposing factors, live alone and neglect their care. 


\section{References}

1. Turhan O, Inan D, Saba R, et al. Rinoserebral mukormikoz: bir olgu sunumu. ANKEM Derg 18 (2004): 228-230.

2. Yen M, Baugh W. Rhinocerebral Mucormycosis [Internet],[Place unknown]: Medline Avaliable from: left angle bracket (2011).

3. Goldstein EJ, Spellberg B, Walsh TJ, et al. Recent advances in the management of mucormycosis: from bench to bedside. Clinical Infectious Diseases 48 (2009): 1743-1751.

4. Solano T, Atkins B, Tambosis E, et al. Disseminated mucormycosis due to Saksenaea vasiformis in an immunocompetent adult. Clin Infect Dis 30 (2000): 942-943.

5. Pinto ME. Hyperglycemic hyperosmolar state and rhino-orbital mucormycosis. Diabetes Res Clin Pract 91 (2011): e37-e39.

6. Parfrey NA. Improved diagnosis and prognosis of mucormycosis. A clinicopathologic study of 33 cases. Medicine (Baltimore) 65 (1986): 113-123.

7. Lee FY, Mossad SB, Adal KA. Pulmonary mucormycosis: the last 30 years. Arch Intern Med 159 (1999): 1301-1309.

8. Buchta $\mathrm{V}$, Kalous $\mathrm{P}$, Otčenášek $\mathrm{M}$, et al. Primary cutaneous Absidia corymbifera infection in a premature newborn. Infection 31 (2003): 57-59.

9. Goel S, Palaskar S, Shetty VP, et al. Rhinomaxillary mucormycosis with cerebral extension. Journal of oral and maxillofacial pathology: JOMFP 13 (2009): 14.

10. Pagano L, Ricci P, Tonso A, et al. Mucormycosis in patients with haematological malignancies: a retrospective clinical study of 37 cases. British journal of haematology 99 (1997): 331-336.

11. Saydam L, Erpek G, Kizilay A. Calcified Mucor fungus ball of sphenoid sinus: an unusual presentation of sinoorbital mucormycosis. Annals of Otology, Rhinology \& Laryngology 106 (1997): 875-877.

12. Mizutari K, Nishimoto K, Ono T. Cutaneous mucormycosis. The Journal of dermatology 26 (1999): 174177.

13. Fu KA, Nguyen PL, Sanossian N. Basilar Artery Territory Stroke Secondary to Invasive Fungal Sphenoid Sinusitis: A Case Report and Review of the Literature. Case Reports in Neurology 7 (2015): 51-58.

14. Kumar NS, Padala RK, Tirupati S, et al. Rhinocerebral Mucormycosis with Top of Basilar Artery Syndrome. J Stroke Cerebrovasc Dis 25 (2016): 378-382.

(C) (9) ${ }_{\text {BY }}^{\text {This article is an open access article distributed under the terms and conditions of the }}$ 